Pacific Journal of Mathematics

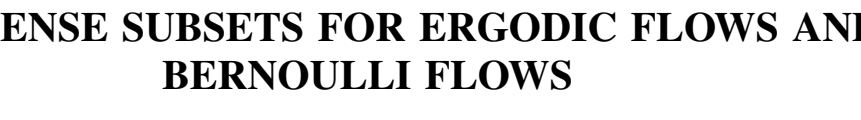




\title{
NICE DENSE SUBSETS FOR ERGODIC FLOWS AND BERNOULLI FLOWS
}

\author{
KYEWON KOH PARK
}

\begin{abstract}
Flows built under step functions are shown to be dense in all ergodic flows in the $\bar{d}$-metric. In particular Bernoulli flows built under step functions with multi-step Markov partitions on their bases make a $\bar{d}$-dense subset for all Bernoulli flows.
\end{abstract}

0. Introduction. Given a stationary process, we approximate this by an $n$-step Markov process that is the most random of the class which has the same joint distribution of $n$-names. We call this the canonical $n$-step Markov approximation. The term "approximation" is in the sense that the process is close in entropy and distribution, which is called the vague topology. D. S. Ornstein [4] proved that the set of Bernoulli processes is the closure in the $\bar{d}$-metric of the set of mixing Markov processes by showing that canonical approximations are mixing and they do converge in the $\bar{d}$-metric. One of the main results in this paper is to find a nice dense subset which characterizes in the same way the Bernoulli flows.

As for ergodic flows in this line, there are two main theorems. One of them is Ambrose's representation theorem [1], which states that any measurable ergodic flow can be represented as a flow built under a function. The most significant part of his theorem is that the $\sigma$-algebra of measurable sets in the representation is the complete product algebra of the measurable sets in the base and the Lebesgue sets in the time axis. The other theorem is by D. S. Ornstein [3] which states that all Bernoulli flows of the same entropy are isomorphic to one another and in particular isomorphic to a flow built under a step function whose values are irrationally related with an independent partition on the base [see Shields, 7].

Later D. Rudolph [6] proved that we can make the function in Ambrose's representation theorem to have two irrationally related values, say a step function. He also showed that if the entropy of a flow is small enough, then we can construct a "natural" partition under the function to generate the $\sigma$-algebra of the flow by making the "natural" base partition generate the base $\sigma$-algebra. 
In the first section we show that every flow has a factor which is built under a step function and is close to itself in the $\bar{d}$-metric. Given a flow under a step function, we approximate the base process by multi-step Markov processes in the sense of distribution and entropy. A flow built under the same step function with an $n$-step Markov approximation on the base is called a canonical approximation of the given flow. It is shown that a flow built under a step function with a multi-step Markov partition on the base is a direct product of two factors, a Bernoulli factor and a rotation factor [5]. We show that if a Bernoulli flow is built under a step function, then the canonical $n$-step Markov approximations are Bernoulli. Hence they do converge in the $\bar{d}$-metric [4].

We always assume that every flow $\left(S_{t}, \bar{P}, \Omega\right)$ is ergodic and is built under a function $f$. Let $\bar{P}=\left\{\bar{P}_{1}, \ldots, \bar{P}_{n}\right\}$ be a partition of a flow built under the function which is bounded below and above (see Ambrose [1]). Since any partition of infinitely many sets can be approximated in the $\bar{d}$-metric by partitions of finitely many sets and we are interested in $\bar{d}$-dense subsets, we may assume that a partition has finitely many sets. A partition $\bar{P}$ is called straight if for every point $x$ in the base, $\left(x, t_{0}\right) \in \bar{P}_{i}$ for some $t_{0}$ implies $(x, t) \in P_{i}$ for all $0 \leq t<f(x)$. If the partition $\bar{P}$ of a flow is straight, we denote the base with the "corresponding" partition by $(T, P, X)$. When a flow $\left(S_{t}, \bar{P}, \Omega\right)$ is built under a step function, the partition $\bar{P}$ always denotes the straight partition. And if the corresponding base process $(T, P, X)$ is multi-step Markov, then we say that the flow $\left(S_{t}, \bar{P}, \Omega\right)$ is built under a step function with a multi-step Markov partition on the base. For any pair $\{\bar{P}, \bar{Q}\}$ of partitions, $|\bar{P}-\bar{Q}|$ denotes $\frac{1}{2}\left(\sum \bar{m}\left(\bar{P}_{i}-\bar{Q}_{i}\right)+\sum \bar{m}\left(Q_{i}-\bar{P}_{i}\right)\right)$. Let $m$ be the measure on $X$ corresponding to $\bar{m}$ on $\Omega$.

By a continuous (flow) $\bar{P}$-name of a point $x$ in $\left(S_{t}, \bar{P}, \Omega\right)$, we mean the line $R$ partitioned in such a way that $t$ in $R$ is in the $i$ th atom of this partition if $S_{t}(x)$ is in the $i$ th atom of $\bar{P}$. By a continuous $\bar{P}$-name of a point $x$ of length $l$, we mean an interval $(o, l)$ partitioned the same way as above.

I would like to thank Professor D. S. Ornstein under whose guidance this work was done and Professor D. Rudolph for his helpful suggestions.

1. A dense subset of ergodic flows. The main result is Theorem 1. However Corollary 1 is stated in more general terms. To prove the theorem, we need the following lemmas.

LEMMA 1. For every flow $\left(S_{t}, \bar{P}, \Omega\right)$ and $\varepsilon>0$, there is a factor $\left(S_{t}, \bar{P}^{\prime}, \Omega\right)$ such that

(i) $\left|\bar{P}-\bar{P}^{\prime}\right|<\varepsilon$

(ii) $\bar{P}^{\prime}$ is a straight partition. 
Proof. This is straight measure theory. Let $\bar{P}=\left\{\bar{P}_{1}, \ldots, \bar{P}_{n}\right\}$. Since the $\sigma$-algebra in $\Omega$ is the complete product $\sigma$-algebra, there exists a collection of measurable rectangles $\left\{R_{i j}\right\}$, whose time axes are intervals such that

(i) $m\left(\bigcup_{j=1}^{l(i)} R_{i j} \Delta P_{i}\right)<\frac{\varepsilon}{100 n} i=1, \ldots, n$,

(ii) $\left\{R_{i j}\right\}$ disjoint,

(iii) $\bigcup_{i, j} R_{i j} \subset \Omega$.

Order the rectangles $\left\{R_{i j}\right\}$ according to the length of time axes and rename them as $\left\{R_{i}\right\}$. Then there exists $k$ such that $m\left(\cup_{i=1}^{k} R_{i}\right)>1-$ $\varepsilon / 100$. We only consider $R_{i j}$ 's in this collection $\left\{R_{i}\right\}_{i=1}^{k}$. Hence we may assume that the lengths of time axes of the collection $\left\{R_{i j}\right\}$ are bounded away from 0 . Let $R_{i j}^{\prime}=R_{i j} \cup\left\{w \mid w \in \Omega-\bigcup_{u, v} R_{u v}, S_{-t_{0}-\delta}(w) \in R_{i j}\right.$ for any small $\delta>0$ \} for all $i$ and $j$ where

$$
t_{0}=\inf \left\{t \mid S_{-t}(w) \in \bigcup_{u, v} R_{u v}\right\} .
$$

Then $\left\{R_{i j}^{\prime}\right\}$ is a collection of measurable sets whose union covers the whole space $\Omega$. Let $\bar{P}_{l}^{\prime}=\bigcup_{j=1}^{\prime(i)} R_{i j}^{\prime}$. It is clear that the partition $\bar{P}^{\prime}$ is a straight partition satisfying $\left|\bar{P}-\bar{P}^{\prime}\right|<\varepsilon$.

LemMa 2. Let $\left(S_{t}, \bar{P}, \Omega\right)$ be a flow with a straight partition. For given $\varepsilon>0$, there exists a factor $\left(S_{t}, \bar{P}^{\prime}, \Omega\right)$ built under a step function satisfying $\left|\bar{P}-\bar{P}^{\prime}\right|<\varepsilon$.

Proof. We will follow the method used by D. Rudolph [6]. Hence, we will be sketchy in some of the details and refer the reader to this paper, in particular the first four pages in checking the measurability of sets. We will just highlight different aspects of the construction. We may assume $m(X)=1$. We will accomplish this by a "successive approximation" method on a proper sub $\sigma$-algebra to ensure the measurability of sets. This sub $\sigma$-algebra consists of all measurable sets $M$ in $\Omega$ with the property that for any measurable function $f: X \rightarrow R$, the set $\{x \mid(x, f(x)) \in M\}$ is a

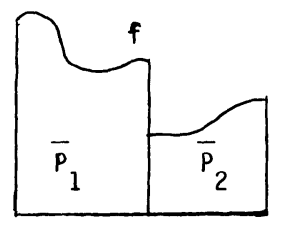

$\left(s_{t}, \bar{P}\right)$
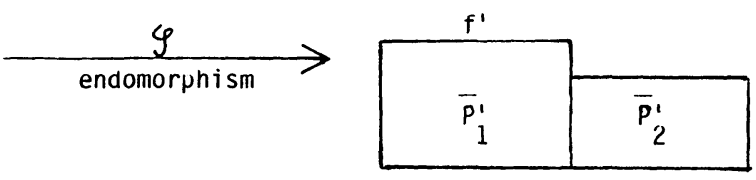

$\left(s_{t}, \bar{p}^{\prime}\right)$ 
measurable subset of $X$. Clearly every set in the partition $\bar{P}$ is in this sub $\sigma$-algebra (for details, see [6]).

Let $\varepsilon_{i}$ be such that $\sum \varepsilon_{i}<\varepsilon$. To avoid complicated notation, we assume that the partition $\bar{P}$ consists of two sets $\left\{\bar{P}_{0}, \bar{P}_{1}\right\}$. Figure I illustrates the lemma in this case.

We first construct $\bar{P}^{(1)}=\left\{\bar{P}_{0}^{(1)}, \bar{P}_{1}^{(1)}\right\}$ such that

(i) $\bar{P}^{(1)}$ is a straight partition

(ii) $\left|\bar{P}^{(1)}-\bar{P}\right|<\varepsilon_{1}$

(iii) $\bar{P}^{(1)}$ is built under an almost step function.

And we will describe how to construct $\bar{P}^{(i+1)}$ from $\bar{P}^{(i)}$ such that

(i) $\bar{P}^{(i+1)}$ is a straight partition

(ii) $\left|\bar{P}^{(i+1)}-\bar{P}^{(i)}\right|<\varepsilon_{i+1}$

(iii) $\overline{\boldsymbol{P}}^{(i+1)}$ is built under a function which is closer to the step function than the one at the previous stage.

Let $\{p, q\}$ be irrationally related $(p<q)$ numbers. Let

$$
\begin{aligned}
& \alpha=\inf \{f(x) \mid x \in X\}, \\
& \beta=\sup \{f(x) \mid x \in X\},
\end{aligned}
$$

$g(\varepsilon)=\min \left\{t \mid s>t \Rightarrow\right.$ there exist $m$ and $n$ in $\mathbf{Z}^{+}$such that

$$
0<s-(n p+m q)<\varepsilon\} \text {. }
$$

Note that $g(\varepsilon)$ is finite for all $\varepsilon>0$.

(Construction of $\bar{P}^{(1)}$ ). Take $n_{1}$ such that

$$
\frac{g\left(\varepsilon_{2} / 3\right)}{n_{1} \cdot \alpha}<\frac{\varepsilon_{2}}{10}
$$

(this is needed for the construction of $\bar{P}^{(2)}$ ). Take an $n_{1}$-long Rochlin tower of the base $X$. Exhaust all the error set by allowing the tower to be longer than $n_{1}$, but $<2 n_{1}$ and wider. Denote the base set of this tower by $F_{1}$. Partition $F_{1}$ into $\left\{F_{1, j}\right\}$ such that

(i) Each atom has the same base $P$-name along the tower.

(ii) For any points $x, y$ in $F_{1, j}$, they satisfy

$$
\left|\sum_{i=0}^{k} f\left(T^{i}(x)\right)-\sum_{i=0}^{k} f\left(T^{i}(y)\right)\right|<\frac{p}{2 \cdot M}
$$

for all $k=0, \ldots, n_{1}-1, n_{1}, \ldots, h^{(1)}(x)-1$ where $M$ satisfies $q / M<\varepsilon_{1} / 2$ and $h^{(1)}(x)$ denotes the height of the base tower of $x$.

$h^{(1)}(x)$ is an integer between $n_{1}$ and $2 n_{1}$. Clearly $F_{1, j}$ 's are measurable. Build the flow tower on each of the $F_{1, j}$ 's. We note from the construction of the $F_{1, j}$ 's that any two points on the same $F_{1, j}$ have almost 
identical flow names up to the end of the tower. Each flow tower has length at least $n_{1} \cdot \alpha$.

Repartition the flow tower built over $F_{1, j}$ with two lengths $\{p / M, q / M\}$ along the time axis except the very top in such a way that the new partition over a base $F_{1, j}$ is different from the given partition $\bar{P}$ by not more than $\varepsilon_{1} / 2$. This is possible because of (ii). We call the blocks of length $p / M$ or $q / M$ good blocks. Let $\bar{P}_{0}^{(1)}$ be the union of $q / M$-long blocks together with the very top if it lies right above $q / M$-long blocks. Let $\bar{P}_{1}^{(1)}$ be the union of $p / M$-long blocks together with the very top if it lies right above a $p / M$-long block. This gives a partition $\bar{P}^{(1)}$ of the flow tower over $F_{1, j}$ satisfying $\left|\bar{P}-\bar{P}^{(1)}\right|<p / 2 M \cdot h^{(1)}(x) \cdot m\left(F_{1, j}\right)<p / 2 M$. $2 n_{1} \cdot m\left(F_{1, j}\right)$. The shaded area in the following Figure II is where the two partitions differ. If we repeat this on each flow tower built over $\left\{F_{1, j}\right\}$, then on $\Omega$ we get

$$
\begin{aligned}
\left|\bar{P}-\bar{P}^{(1)}\right| & <\sum_{j} \frac{p}{2 M} \cdot 2 n_{1} \cdot m\left(F_{1, j}\right)=\frac{p}{2 M} \cdot 2 n_{1} \cdot \sum_{j} m\left(F_{1, j}\right) \\
& =\frac{p}{2 M} 2 n_{1} \cdot m F_{1} \leq \frac{p}{2 M} 2 n_{1} \cdot \frac{1}{n_{1}}=\frac{p}{M}<\varepsilon .
\end{aligned}
$$

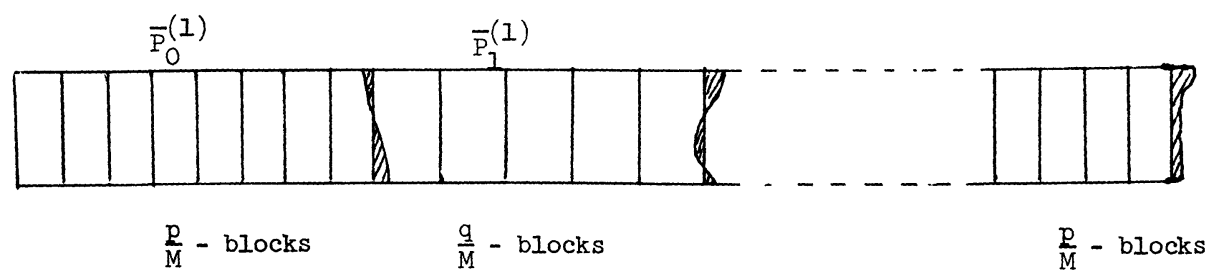

FIGURE II

The flow with the partition $\bar{P}^{(1)}$ will look like the following.

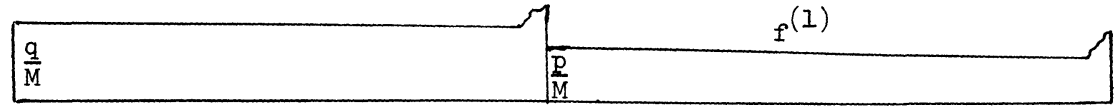

$\left.{ }_{(T}^{(1)}, P^{(1)}, X^{(1)}\right)$

Figure III

Denote the base by $\left(T^{(1)}, P^{(1)}, X^{(1)}\right)$ and the function by $f^{(1)}$.

(Construction $\bar{P}^{(i+1)}$ from $\left.\bar{P}^{(2)}\right)$. By choosing $\varepsilon_{i}$ 's small enough, we may assume that $\sum_{i=1}^{\infty} \varepsilon_{i} /(\alpha-\varepsilon)<\varepsilon$. Consider the flow tower built over $F_{i}$. We denote by $h^{(i)}$ the height of the base tower at the $i$ th stage. We define 
a new partition $\bar{P}^{(i+1)}$ in such a way that the new names on the top or bottom ends of the tower of length at most $\varepsilon_{i+1}(p / M) \cdot h^{(i)}$ are defined very differently but the new names on the center section of length at least $\left(1-3 \varepsilon_{i+1}\right)(p / M) \cdot h^{(i)}$ are shifted by at most $\varepsilon_{i+1}$. Take $n_{i+1}$ such that

$$
\begin{aligned}
& \text { (1) } \frac{(q / M) \cdot n_{i}}{(p / M) \cdot n_{i+1}}<\frac{\varepsilon_{i+1}}{10} \\
& \text { (2) } \frac{g\left(\varepsilon_{i+2} / 3\right)}{(p / M) \cdot n_{i+1}}<\frac{\varepsilon_{i+2}}{10} \\
& \text { (3) } \frac{m\left(X^{(i)}\right)}{n_{i+1}} \cdot \frac{q}{M}<\varepsilon_{i+1} .
\end{aligned}
$$

Build an $n_{i+1}$-long Rochlin tower of $\left(T^{(i)}, X^{(i)}\right)$. To avoid complications of notation, we will write $T$ for $T^{(i)}$ in this section. Allow the tower to be longer but $<2 n_{i+1}$ and wider to exhaust all the error set. Let the base be $F_{t+1}$. Divide $F_{i+1}$ into $\left\{F_{i+1, j}\right\}$ such that

(i) They have the same base $P^{(i)}$ name along the tower.

(ii) For any points $x, y \in F_{i+1, j}$, they satisfy

$$
\begin{aligned}
& \left|\sum_{d=0}^{k} f^{(i)}\left(T^{d}(x)\right)-\sum_{d=0}^{k} f^{(i)}\left(T^{d}(y)\right)\right| \\
& <\frac{\varepsilon_{i+1}}{3} \quad \text { for all } k=0, \ldots, h^{(i+1)}(x)-1 .
\end{aligned}
$$

That is to say, on each $F_{l+1, j}$, they have nearly identical flow $\overline{\boldsymbol{P}}^{(i)}$-names up the tower. Again it is clear that the flow towers built over $F_{i+1, j}$ 's are measurable. We redefine the flow name on this tower as follows: Consider the first $l$ such that $f^{(i)}\left(T^{l}(x)\right) \neq p / M$ or $q / M$ for some $x \in F_{i+1, j}$. Let $l^{\prime}$ be the first number after $l$ such that $f^{(i)}\left(T^{l^{\prime}+1}(x)\right)=p / M$ or $q / M$ for all $x \in F_{\imath+1, j}$. We will call the set

$$
\left\{(x, t) \mid x \in F_{i+1, j}, \sum_{k=0}^{l-1} f^{(l)}\left(T^{k}(x)\right) \leq t<\sum_{k=0}^{l^{\prime}} f^{(i)}\left(T^{k}(x)\right)\right\}
$$

a bad set.

We may assume each bad set occurs at the same time on $F_{i+1, j}$ (otherwise, we divide $F_{i+1, j}$ into a finer partition so that this is the case). From the construction, this bad set always lies either below or above an $n_{i}$-long string of good blocks,

$\left(\mathrm{C}_{1}\right)$ for all $x \in F_{i+1, j}$

$$
\left[\sum_{k=0}^{l-1} f^{(i)}\left(T^{k}(x)\right)-g\left(\frac{\varepsilon_{i+1}}{3}\right)-\left(\frac{p}{M}+\frac{q}{M}\right), \sum_{k=0}^{l-1} f^{(i)}\left(T^{k}(x)\right)\right]
$$


is a sequence of good blocks or

$\left(\mathrm{C}_{2}\right)$ for all $x \in F_{i+1, j}$

$$
\left[\sum_{k=0}^{l^{\prime}} f^{(i)}\left(T^{k}(x)\right), \sum_{k=0}^{l-1} f^{(i)}\left(T^{k}(x)\right)+g\left(\frac{\varepsilon_{i+1}}{3}\right)+\left(\frac{p}{M}+\frac{q}{M}\right)\right]
$$

is a sequence of good blocks.

We consider the first case where a sequence of good blocks lies below the bad set. Let $k_{0}$ be an integer such that

$$
\begin{aligned}
\sum_{k=0}^{l-1} f^{(i)}\left(T^{k}(x)\right)-g\left(\frac{\varepsilon_{i+1}}{3}\right)-\left(\frac{p}{M}+\frac{q}{M}\right) & <\sum_{k=0}^{k_{0}} f^{(i)}\left(T^{k}(x)\right) \\
& <\sum_{k=0}^{l-1} f^{(i)}\left(T^{k}(x)\right)-g\left(\frac{\varepsilon_{i+1}}{3}\right) .
\end{aligned}
$$

Then we have

$$
g\left(\frac{\varepsilon_{i+1}}{3}\right)<\sum_{k=k_{0}+1}^{l-1} f^{(i)}\left(T^{k}(x)\right)<g\left(\frac{\varepsilon_{i+1}}{3}\right)+\frac{p}{M}+\frac{q}{M} .
$$

We know that there exist integers $n_{p}$ and $n_{q}$ with

$0 \leq \sum_{k=k_{0}+1}^{l^{\prime}} f^{(i)}\left(T^{k}(x)\right)-\left(n_{p} \frac{p}{M}+n_{q} \frac{q}{M}\right)<\frac{2 \cdot \varepsilon_{l+1}}{3}$ for all $x \in F_{i+1, j}$.

On $\cup S_{t}(x)$ where

$$
\sum_{k=0}^{k_{0}} f^{(i)}\left(T^{k}(x)\right) \leq t \leq \sum_{k=0}^{k_{0}} f^{(i)}\left(T^{k}(x)\right)+n_{p} \cdot \frac{p}{M}+n_{q} \cdot \frac{q}{M},
$$

we define

$$
\begin{gathered}
\bar{P}_{0}^{(i+1)=} \bigcup_{x \in F_{1+1, j}} \bigcup_{t_{0} \leq t<t_{1}} S_{t}(x) \quad \text { where } t_{0}=\sum_{k=0}^{k_{0}} f^{(i)}\left(T^{k}(x)\right) \\
\text { and } t_{1}=t_{0}+n_{p} \cdot \frac{p}{M} \\
\bar{P}_{1}^{(i+1)}=\bigcup_{x \in F_{i+1, J}} \bigcup_{t_{1} \leq t<t_{2}} S_{t}(x) \quad \text { where } t_{2}=t_{1}+n_{q} \cdot \frac{q}{M} .
\end{gathered}
$$

Shift the next string of good blocks down on top of this newly defined good block. The names are shifted by at most $\left(2 \cdot \varepsilon_{i+1}\right) / 3$. We call the blocks that have to be shifted by at most $2 \cdot \varepsilon_{i+1} / 3$ very good blocks. The center section whose length is bigger than $n_{i}(p / M)\left(1-3 \varepsilon_{i+1}\right)$ is a string 
of very good blocks. The new $\overline{\boldsymbol{P}}^{(i+1)}$ names of the end sections of length at most $n_{i}(p / M) \varepsilon_{i+1}$ (by (2)) may differ a lot from $\bar{P}^{(i)}$ names. This gives a good continuous name to a point $x \in F_{i+1, j}$ for $t \in\left[0, \sum_{k=0}^{l_{0}-1} f^{(i)}\left(T^{k}(x)\right)\right]$ where $l_{0}$ is the first next number $>l^{\prime}$ satisfying $f^{(i)}\left(T^{l_{0}}(x)\right) \neq p / M$ or $q / M$ for some $x \in F_{i+1, j}$. In the case of $\left(\mathrm{C}_{2}\right)$, we choose $g\left(\varepsilon_{i+1} / 3\right)+$ $p / M+q / M$-long blocks above the bad set. We define $\bar{P}^{(i+1)}$ as we did before on this section which may differ a lot from $\bar{P}^{(i)}$ and we shift good blocks down by at most $2 \varepsilon_{i+1} / 3$ to get $\bar{P}^{(i+1)}$. Keep redefining the names whenever a bad set occurs on each $F_{i+1, j}$. This gives a new partition $\bar{P}^{(i+1)}$ on each $F_{\imath+1, j}$ satisfying $\left|\bar{P}^{(i)}-\overline{\boldsymbol{P}}^{(i+1)}\right|<\varepsilon_{i+1}$. Hence $\left|\overline{\boldsymbol{P}}^{(i)}-\overline{\boldsymbol{P}}^{(i+1)}\right|<\varepsilon_{i+1}$ on $\Omega$.

Except a set of measure less than $\varepsilon_{i+1}$ every point is contained in one of the good blocks with respect to the partition $\bar{P}^{(i+1)}$ by (3).

Let $\bar{P}^{\prime}=\lim _{i \rightarrow \infty} \bar{P}^{(i)}$. Clearly $\left|\bar{P}-\bar{P}^{\prime}\right|<\varepsilon$. Using the Borel-Cantelli lemma, we see that except for a set of measure zero, the continuous $\bar{P}^{\prime}$-name of a point in $\Omega$ is a sequence of length $p / M$ or $q / M$. For this, see D. Rudolph [6].

To complete the proof of the lemma we have to show that the measure in the space with the new partition $\bar{P}^{\prime}$ is properly defined. For this we use the theorem of Amrose, whose proof will be omitted (see Ambrose [1]).

THEOREM. Let $\left(S_{t}, \Omega\right)$ be an ergodic flow built under a function $f$, with measure $\bar{m}$. If the functions $F(w)=F(x, t)=f(x)$ and $G(w)=G(x, t)=t$ are measurable, then there exists a measure $m$ on $X$ for which $f$ is $a$ measurable function and $T$ is a measure preserving transformation such that $\bar{m}$ is the complete direct product measure of $m$ on $X$ with Lebesgue measure on the time axis.

We have to show $F(w)$ and $G(w)$ are measurable. At the $i$ th stage, let $A_{t}=\left\{w \mid F^{(i)}(w)=F^{(i)}(x, t)=f^{(i)}(x)=p / M\right\}$ and $B_{i}=\left\{w \mid F^{(i)}(w)=\right.$ $\left.F^{(t)}(x, t)=f^{(i)}(x)=q / M\right\}$.

$$
\left\{w: F(w)=\frac{p}{M}\right\}=\bigcup_{k} \bigcap_{i \geq k} A_{\imath} \text { and }\left\{w: F(w)=\frac{q}{M}\right\}=\bigcup_{k} \bigcap_{i \geq k} B_{i} .
$$

Since all of $A_{i}$ 's and $B_{i}$ 's are measurable (see [6]), $\{w: F(w)=p / M\}$ and $\{w: F(w)=q / M\}$ are measurable. Hence the function $F$ is measurable. Similarly $\left\{w \mid G(w)<t_{0}\right\}$ is a countable union of countable intersection of measurable sets for every $t_{0}$. Hence $G$ is also a measurable function. 
TheOREM 1. Every flow $\left(S_{t}, \bar{P}, \Omega\right)$ has a factor $\left(S_{t}, \bar{P}^{\prime}, \Omega\right)$ which is built under a step function satisfying $\left|\bar{P}-\bar{P}^{\prime}\right|<\varepsilon$ for any $\varepsilon>0$.

COROLlaRY 1. Flows built under step functions make a dense subset of all ergodic flows in the $\bar{d}$-metric.

\section{A dense subset of Bernoulli flows.}

LEMMA 3. Every flow built under a step function can be approximated in entropy and distribution by flows under step functions with multi-step Markov partitions on the bases.

Proof. Let $(T, P, X)$ be the base of a given flow $\left(S_{t}, \bar{P}, \Omega\right)$ under a function $f$. Let $\left\{\left(T^{(n)}, P^{(n)}, X\right)\right\}$ be a sequence of canonical $n$-step Markov approximations to $(T, P, X)$. Consider the sequence of flows $\left\{\left(S_{t}^{(n)}, \bar{P}^{(n)}, \Omega\right)\right\}$. Each $\left(S_{t}^{(n)}, \bar{P}^{(n)}, \Omega\right)$ is built under the same step function $f$ with a base $\left(T^{(n)}, P^{(n)}, X\right)$. Clearly these flows converge to $\left(S_{t}, \bar{P}, \Omega\right)$ in entropy and distribution.

Proposition 1. Canonical n-step Markov approximations of a flow $\left(S_{t}, \bar{P}, \Omega\right)$ under a step function are Bernoulli flows if $\left(S_{t}, \bar{P}, \Omega\right)$ is Bernoulli.

Proof. Let $\left(S_{t}^{(n)}, \bar{P}^{(n)}, \Omega\right)$ be a $k$-step Markov approximation. We know that this is a direct product of a Bernoulli factor with a rotation [5]. But this rotation factor has to be spanned by finite continuous names which are identical to that of $\left(S_{t}, P, \Omega\right)$. Since $\left(S_{t}, \bar{P}, \Omega\right)$ doesn't have a rotation factor, this Markov approximation cannot have a rotation factor. Hence $\left(S_{t}^{(n)}, \bar{P}^{(n)}, \Omega\right)$ is Bernoulli for all $n$.

LeMMA 4. Let $\left(S_{t}, \bar{P}, \Omega\right)$ be a flow under a step function $f$ and the corresponding base partition $P$ be a generating partition on the base. Then $\bar{P}$ generates under $S_{\delta}$ if $S_{\delta}$ is ergodic and $0<\delta<\frac{1}{2} \min \{$ values of $f\}$.

Proof. We will prove that $\bar{P}$ separates points under $S_{\delta}$. Since $\delta$ $<\frac{1}{2} \min \{$ values of $f\}$, it's clear that $\left(x, t_{1}\right)$ and $\left(y, t_{2}\right)$ have different names under $S_{\delta}$. For every $t_{1}>t_{2}$ (we may assume $t_{1}-t_{2}<\delta$ ), let $E=\left\{(x, t) \mid T^{-1}(x)\right.$ and $x$ belong to different sets in $P, 0 \leq t<$ $\left.\frac{1}{2}\left(t_{1}-t_{2}\right)\right\}$.

Since the flow is ergodic and $m(E)>0$, by the ergodic theorem $\left(x, t_{1}\right)$ hits the set $E$ under $S_{\delta}$ infinitely often. Everytime it hits the set $E$, $\left(x, t_{2}\right)$ belongs to a different set in $\bar{P}$. Therefore $\left(x, t_{1}\right)$ and $\left(x, t_{2}\right)$ have different $\bar{P}$-names under $S_{\delta}$. 
Proposition 2. If $\left(S_{t}, \bar{P}, \Omega\right)$ is a Bernoulli flow built under a step function, then the canonical n-step Markov approximations converge in $\bar{d}$-metric.

Proof. This is clear from Proposition 1 and Lemma 4.

Theorem 1 and Proposition 2 will prove the following Proposition.

Proposition 3. Every Bernoulli flow can be approximated arbitrarily well in the $\bar{d}$-metric by Bernoulli flows, each built under a step function whose values are irrationally related with a multi-step Markov partition on the base.

Since a $\bar{d}$-limit of Bernoulli flows in Bernoulli (see Ornstein [4]), we have the following theorem from Proposition 3.

THEOREM 2. Bernoulli flows are the closure in the $\bar{d}$-metric of the mixing flows, each built under a step function (whose values are irrationally related) with a multi-step Markov partition on the base.

\section{REFERENCES}

[1] W. Ambrose, Representation of ergodic flows, Ann. of Math., 42, 723-739.

[2] D. S. Ornstein, Bernoulli shifts with the same entropy are isomorphic, Advances in Math., 4 (1970), 337-352.

[3] _ The isomorphism theorem for Bernoulli flows, Advances in Math., 10 (1973), 124-142.

[4] __ Ergodic Theory, Randomness, and Dynamical Systems, Yale University Press.

[5] K. Park, A special family of ergodic flows and their $\bar{d}$-limits, Israel J. Math., 42, No. 4, 343-352.

[6] D. J. Rudolph, A two-valued step coding for ergodic flows, Mathematische Zeitschrift, (1976), 201-220.

[7] P. Shields, The theory of Bernoulli shifts, Chicago University Press, Chicago, 1973.

Received February 3, 1983 and in revised form June 27, 1984.

The OHio State University

Columbus, $\mathrm{OH} 43221$ 


\section{PACIFIC JOURNAL OF MATHEMATICS EDITORS}

\author{
V. S. VARADARAJAN (Managing Editor) \\ University of California \\ Los Angeles, CA 90024 \\ Charles R. DePrima \\ California Institute of Technology \\ Pasadena, CA 91125 \\ R. FINN \\ Stanford University \\ Stanford, CA 94305
}

\author{
HeRmanN FlaschKa \\ University of Arizona \\ Tucson, AZ 85721 \\ Ramesh A. Gangolli \\ University of Washington \\ Seattle, WA 98195 \\ ROBION KIRBY \\ University of California \\ Berkeley, CA 94720
}

C. C. MOORE

University of California

Berkeley, CA 94720

H. SAMELSON

Stanford University

Stanford, CA 94305

HAROLD STARK

University of California, San Diego

La Jolla, CA 92093

ASSOCIATE EDITORS
R. ARENS
E. F. BECKENBACH
B. H. NeUmanN
F. WOLF
K. YosHIDA (1906-1982)

\section{SUPPORTING INSTITUTIONS}

UNIVERSITY OF ARIZONA

UNIVERSITY OF BRITISH COLUMBIA

CALIFORNIA INSTITUTE OF TECHNOLOGY

UNIVERSITY OF CALIFORNIA

MONTANA STATE UNIVERSITY

UNIVERSITY OF NEVADA, RENO

NEW MEXICO STATE UNIVERSITY

OREGON STATE UNIVERSITY
UNIVERSITY OF OREGON

UNIVERSITY OF SOUTHERN CALIFORNIA

STANFORD UNIVERSITY

UNIVERSITY OF HAWAII

UNIVERSITY OF TOKYO

UNIVERSITY OF UTAH

WASHINGTON STATE UNIVERSITY

UNIVERSITY OF WASHINGTON 


\section{Pacific Journal of Mathematics}

Vol. 119, No. $1 \quad$ May, 1985

Maurice Chacron, Nonisotropic unitary spaces and modules with

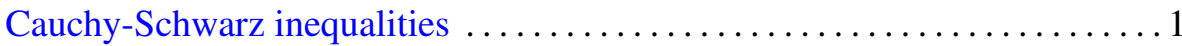

Myriam Dechamps-Gondim, Françoise Piquard and H. Queffélec, On

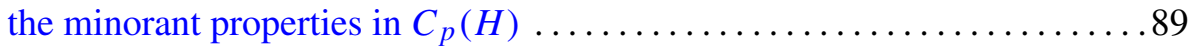

Klaus Floret and V. B. Moscatelli, On bases in strict inductive and projective limits of locally convex spaces .................... 103

Norman Joseph Goldstein, Degenerate secant varieties and a problem on

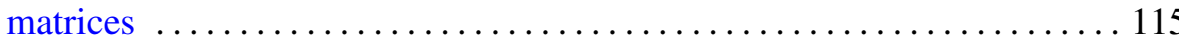

Harold Morris Hastings and Stefan Waner, $G$-bordism with singularities

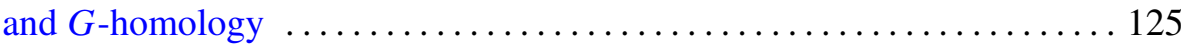

Takesi Isiwata, Clopen realcompactification of a mapping ........... 153

Hisao Kato, Concerning hyperspaces of certain Peano continua and strong

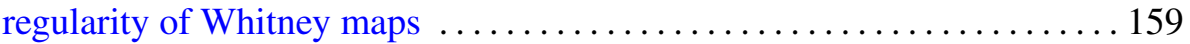

Elyahu Katz and Sidney Allen Morris, Free products of topological groups

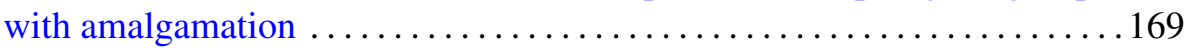

Kyewon Koh Park, Nice dense subsets for ergodic flows and Bernoulli

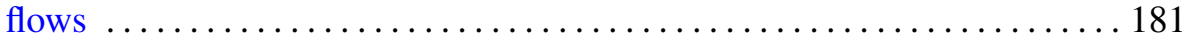

Francis Pastijn and Peter George Trotter, Lattices of completely regular

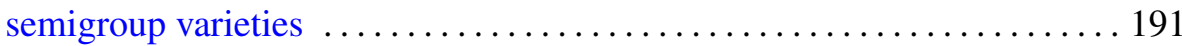

Rae Michael Andrew Shortt, Reticulated sets and the isomorphism of analytic powers

David A. Stegenga and Kenneth R. Stephenson, Generic covering properties for spaces of analytic functions

M. V. Subba Rao and R. Sitaramachandra Rao, On some infinite series of

L. J. Mordell and their analogues 